\title{
Conformational and rheological properties of semiflexible polymers in shear flow
}

Roland G. Winkler

Citation: The Journal of Chemical Physics 133, 164905 (2010); doi: 10.1063/1.3497642

View online: https://doi.org/10.1063/1.3497642

View Table of Contents: http://aip.scitation.org/toc/jcp/133/16

Published by the American Institute of Physics

\section{Articles you may be interested in}

The rheology of dilute solutions of flexible polymers: Progress and problems Journal of Rheology 49, 1 (2005); 10.1122/1.1835336

Dynamics of entangled linear polymer melts: A molecular-dynamics simulation

The Journal of Chemical Physics 92, 5057 (1990); 10.1063/1.458541

Variational Treatment of Hydrodynamic Interaction in Polymers

The Journal of Chemical Physics 50, 4831 (1969); 10.1063/1.1670977

Internal dynamics of semiflexible polymers with active noise

The Journal of Chemical Physics 146, 154903 (2017); 10.1063/1.4981012

Coil-stretch transition of dilute flexible polymers under ultrahigh velocity gradients

The Journal of Chemical Physics 60, 5030 (1974); 10.1063/1.1681018

Dynamics of single semiflexible polymers in dilute solution

The Journal of Chemical Physics 145, 234903 (2016); 10.1063/1.4971861

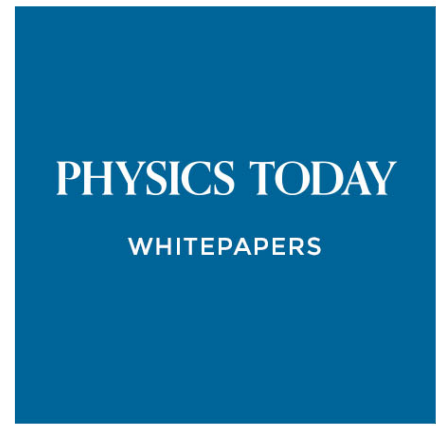




\title{
Conformational and rheological properties of semiflexible polymers in shear flow
}

\author{
Roland G. Winkler ${ }^{\mathrm{a})}$ \\ Institute for Advanced Simulation, Forschungszentrum Jülich, 52428 Jülich, Germany
}

(Received 10 August 2010; accepted 15 September 2010; published online 27 October 2010)

\begin{abstract}
A theoretical description is provided for the nonequilibrium conformational and dynamical properties of a polymer in shear flow. Using a mean-field semiflexible chain model, which accounts for hydrodynamic interactions within the preaveraging approximation, analytical expressions are derived for the dependence of the deformation, orientation, and relaxation times on polymer persistence length and shear rate. Moreover, the rheological properties of a dilute polymer solution are discussed. The model yields shear thinning at large Weissenberg numbers. The analytical results are compared with fluorescence microscopy measurements of individual DNA molecules, which yield qualitative and partial quantitative agreement. (C) 2010 American Institute of Physics.

[doi:10.1063/1.3497642]
\end{abstract}

\section{INTRODUCTION}

Individual polymers in solution exhibit a remarkably reach structural and dynamical behavior in external flow fields. ${ }^{1,2}$ Fluorescence microscopy studies on single DNA molecules in shear flow reveal large conformational changes due to tumbling motion. ${ }^{1-4} \mathrm{~A}$ polymer chain continuously undergoes stretching and compression cycles and never reaches a steady-state extension. The detailed evolution itself depends upon the shear rate. The microscopic conformational and dynamical properties are tightly linked to the macroscopic rheological behavior of a polymer solution and give rise to phenomena such as shear rate dependent viscosities, normal stress differences, and shear thinning. ${ }^{5-7}$

By the same experimental technique, valuable quantitative information has been obtained for the nonequilibrium properties of DNA molecules, such as their deformation, orientation, and viscosity, both, for free and tethered molecules. ${ }^{1,3,4,8-11}$ Moreover, the extension and relaxation behavior have been measured ${ }^{1,10}$ as well as the tumbling time $^{3,4}$ and the orientational distribution function of deformed DNA molecules. ${ }^{4}$

Since shear flows are ubiquitous in biological systems and technical applications, e.g., microfluidics, capillary flow, or flows near surfaces, the understanding of the dynamics of individual flexible and semiflexible polymers, such as DNA, actin filaments, or fd-viruses is of fundamental interest.

Various analytical approaches have been adopted to achieve a microscopic understanding of the nonequilibrium properties of polymers in flow. Often flexible polymers are considered neglecting hydrodynamic and excluded volume interactions, ${ }^{6,7,12-19}$ but taking finite chain extensibility into account. $^{20}$ Other approaches account for excluded volume interactions $^{21,22}$ or take hydrodynamic interactions, ${ }^{23}$ or both, ${ }^{24,25}$ and finite chain extensibility into account. ${ }^{26,27}$ The effect of chain stiffness has been addressed in Refs. 28-30.

${ }^{a)}$ Electronic mail: r.winkler@fz-juelich.de.
A broad spectrum of nonequilibrium computer simulations has been performed to overcome deficiencies of analytical approaches. Brownian dynamics simulations have been performed for Gaussian polymers with ${ }^{31}$ and without ${ }^{32}$ hydrodynamic interactions. More realistic simulations account for the finite chain extensibility and excluded volume interactions. ${ }^{33-36}$ In Brownian dynamics simulations, hydrodynamic interactions are taken into account by the Oseen tensor $^{5,37}$ or the Rotne-Prager tensor. ${ }^{38}$ Aside from Ref. 39, which employs Gaussian polymers, typically finite extensible polymers are considered with excluded volume interactions. ${ }^{3,40-48}$ Moreover, molecular dynamics simulations with explicit solvent have been performed in Refs. 49-51. Results of mesoscale hydrodynamics simulations are presented in Ref. 48 exploiting the lattice Boltzmann method. In addition, the multiparticle collision dynamics approach has been used ${ }^{52,53}$ and the smoothed profile method. ${ }^{54}$

The various simulations yield nonequilibrium properties very similar to the experimentally observed behavior on a polymer length scale, e.g., polymer deformation and alignment along the flow direction as well as polymer tumbling, and on macroscopic scales, e.g., non-Newtonian viscosities due to shear thinning at sufficiently large flow rates. ${ }^{6}$ For the latter, a considerable range of power-law exponents, which describe the decrease of the viscosity with shear rate, has been found and there seems to be no consensus on the asymptotic dependence in the limit of an infinite shear rate. There are several reasons for the observed dependencies, which are sometimes related to the particular approach. However, often the range of considered polymer lengths and shear rates is too small. Thus, rather a crossover behavior is observed than the asymptotic behavior.

Despite certain shortcomings, the analytical calculations and computer simulations provide valuable insight into the nonequilibrium behavior of polymers. However, for a microscopic understanding of the flow phenomena an analytical description is desirable, specifically an approach that quantitatively accounts for experimental findings. Such a compre- 
hensive description seems to be difficult to achieve, because of the complexity of interactions such as inextensible polymer bonds, excluded volume and hydrodynamic interactions, and chain stiffness. However, computer simulations ${ }^{3,9}$ and theoretical studies ${ }^{21}$ suggest that excluded volume interactions are of minor relevance at large shear rates and that the conformational, dynamical, and rheological properties are rather generic features of polymers in flow.

Experiments $^{55-57}$ and computer simulations ${ }^{58,59}$ have shown that the equilibrium dynamical properties of semiflexible DNA molecules in solution are quantitatively described by a Gaussian semiflexible polymer model ${ }^{60-67}$ over a wide range of flexibilities. This model has also been used to successfully describe the orientational distribution and tumbling dynamics of DNA molecules in shear flow. ${ }^{29}$ In the present article, the same model is applied to study the conformational, dynamical, and rheological properties of polymers. Various quantities are compared with experimental results on DNA molecules. Moreover, the influence of stiffness is discussed in detail, specifically for objects much stiffer than $\lambda$-DNA.

The studies reach beyond those of Ref. 28, where the Harris-Hearst model ${ }^{68}$ is adopted to describe semiflexible polymers under shear, in several respects, specifically hydrodynamic interactions are taken into account by a preaveraged hydrodynamic tensor. I like to stress that the Harris-Hearst model does not correctly describe the equilibrium properties of a semiflexible polymer, ${ }^{63,69}$ but an adequate description of flexible polymers can be expected.

The paper is organized is follows. In Sec. II, the theoretical model of the semiflexible polymer is outlined. Section III presents results for conformational and dynamical properties, and in Sec. IV the rheological characteristics are discussed. Results are compared with experimental data in Sec. V. Finally, Sec. VI summarizes the major findings.

\section{MODEL: SEMIFLEXIBLE POLYMER IN SHEAR FLOW}

\section{A. Equations of motion}

A mean-field model is adopted for a semiflexible polymer, ${ }^{60-65}$ which is denoted as Gaussian semiflexible polymer. Here, the polymer is considered as a continuous, differentiable space curve $\boldsymbol{r}(s, t)$, where $s(-L / 2 \leq s \leq L / 2)$ is the contour coordinate along the chain of length $L$ and $t$ is the time. In the presence of hydrodynamic interactions, ${ }^{5,37}$ the equation of motion is given by the Langevin equation $^{29,56,67,70,71}$

$$
\begin{aligned}
\frac{\partial}{\partial t} \boldsymbol{r}(s, t)= & \int_{-L / 2}^{L / 2} \mathbf{H}\left(\boldsymbol{r}(s), \boldsymbol{r}\left(s^{\prime}\right)\right)\left[2 \nu k_{B} T \frac{\partial^{2}}{\partial s^{\prime 2}} \boldsymbol{r}\left(s^{\prime}, t\right)\right. \\
& \left.-\epsilon k_{B} T \frac{\partial^{4}}{\partial s^{\prime 4}} \boldsymbol{r}\left(s^{\prime}, t\right)+\boldsymbol{\Gamma}\left(s^{\prime}, t\right)\right] d s^{\prime}+\mathbf{K} \boldsymbol{r}(s, t),
\end{aligned}
$$

with the boundary conditions

$$
\left[2 \nu \frac{\partial}{\partial s} \boldsymbol{r}(s, t)-\epsilon \frac{\partial^{3}}{\partial s^{3}} \boldsymbol{r}(s, t)\right]_{ \pm L / 2}=0,
$$

$$
\left[2 \nu_{0} \frac{\partial}{\partial s} \boldsymbol{r}(s, t) \pm \epsilon \frac{\partial^{2}}{\partial s^{2}} \boldsymbol{r}(s, t)\right]_{ \pm L / 2}=0 .
$$

The stochastic force $\boldsymbol{\Gamma}(s, t)$ is assumed to be stationary, Markovian, and Gaussian with zero mean. ${ }^{5,72} T$ is the temperature and $k_{B}$ is the Boltzmann constant. The hydrodynamic tensor is defined as $\mathbf{H}\left(\boldsymbol{r}(s), \boldsymbol{r}\left(s^{\prime}\right)\right)=\mathbf{Q}\left(\boldsymbol{r}(s)-\boldsymbol{r}\left(s^{\prime}\right)\right)$ $+\mathbf{I} \delta\left(s-s^{\prime}\right) / 3 \pi \eta$, where the term with the delta function accounts for the local friction, $\mathbf{Q}\left(\boldsymbol{r}(s)-\boldsymbol{r}\left(s^{\prime}\right)\right)$ is the RotnePrager tensor ${ }^{29,38,56,67,71}$ and $\eta$ the solvent viscosity. The term with the second derivative captures the chain flexibility, i.e., it accounts for the chain entropy. The term with the fourth derivative is the bending force. Only the element $K_{x y}=\dot{\gamma}$ of the shear rate tensor $\mathbf{K}$ is different from zero, where $\dot{\gamma}$ is the shear rate. The Lagrangian multipliers $\nu(s), \nu_{0}=\nu( \pm L / 2)$, and $\epsilon$ are determined by constraints. ${ }^{73}$ In general, this yields for a polymer in three dimensions $\epsilon=3 /(4 p)$ and $\nu_{0}=3 / 4$, where $p$ is related to the persistence length $l_{p}$ via $p=1 /\left(2 l_{p}\right) .{ }^{65,73}$ The Lagrangian multiplier $\nu(s)$ is determined by the condition $\left\langle\boldsymbol{u}(s)^{2}\right\rangle=1$, with the tangent vector $\boldsymbol{u}(s)$ $=\partial \boldsymbol{r} / \partial s$, and depends on shear rate. Under nonequilibrium conditions, the tension varies along the polymer contour. Hence, $\nu$ depends on $s$ and has to be determined selfconsistently. To avoid this complication, the local constraints are relaxed and replaced by the global one

$$
\int_{-L / 2}^{L / 2}\left\langle\boldsymbol{u}^{2}\right\rangle d s=L
$$

As a consequence, the polymer will be stronger stretched in the central part and less toward its ends. ${ }^{65}$ However, the full solution of a discrete free draining polymer model with individual Lagrangian multipliers for every bond and bond angle ${ }^{63,65,73}$ respectively, yields expectation values for global quantities such as viscosity that deviate only very little from those determined with the constraint (4) in the limit of a nearly continuous polymer. Hence, the solution of the equations of motion with the constraint (4) suffices for any practical purpose.

The mean-field character of the model is reflected in the particular persistence length dependence of $\epsilon$ and the average constraint for the tangent vector, which implies nonzero contour length fluctuations. The Kratky-Porod wormlike chain model $^{74-76}$ requires $\boldsymbol{u}(s)^{2}=1$ and leads to a different $\epsilon^{71,73}$

To solve the nonlinear equations of motion, Zimm's preaveraging approximation is applied, ${ }^{5,56,77}$ which leads to the preaveraged hydrodynamic tensor $\mathbf{H}\left(s, s^{\prime}\right)$ $=\mathbf{I}\left[\delta\left(s-s^{\prime}\right) / 3 \pi \eta+Q\left(s-s^{\prime}\right)\right]$, with

$$
Q(s)=\frac{\Theta(|s|-d)}{3 \pi \eta} \sqrt{\frac{3}{2 \pi \sigma(s)}} \exp \left(-\frac{3 d^{2}}{2 \sigma(s)}\right) .
$$

The Heaviside step function $\Theta$ is introduced to exclude selfinteractions and $d$ is the thickness of the molecule. ${ }^{56,67,71} \sigma$ $=\left\langle\left(\boldsymbol{r}(s)-\boldsymbol{r}\left(s^{\prime}\right)\right)^{2}\right\rangle$ is the mean square distance between two points at $s$ and $s^{\prime}$ 


$$
\sigma(s)=\frac{|s|}{p}-\frac{1}{2 p^{2}}[1-\exp (-2 p|s|)] .
$$

The linear equations of motion are solved by the eigenfunction expansion ${ }^{5,56,66,67,71}$

$$
\boldsymbol{r}(s, t)=\sum_{n=0}^{\infty} \boldsymbol{\chi}_{n}(t) \psi_{n}(s), \quad \boldsymbol{\Gamma}(s, t)=\sum_{n=0}^{\infty} \boldsymbol{\Gamma}_{n}(t) \psi_{n}(s),
$$

where $\psi_{n}(s)$ satisfies the eigenvalue equation

$$
\epsilon k_{B} T \frac{d^{4}}{d s^{4}} \psi_{n}(s)-2 \nu k_{B} T \frac{d^{2}}{d s^{2}} \psi_{n}(s)=\xi_{n} \psi_{n}(s),
$$

with the eigenvalue $\xi_{n}$. The eigenfunctions are given by

$$
\begin{aligned}
& \psi_{0}=\sqrt{\frac{1}{L}}, \\
& \psi_{n}(s)=\sqrt{\frac{c_{n}}{L}}\left(\zeta_{n}^{\prime} \frac{\sinh \zeta_{n}^{\prime} s}{\cosh \zeta_{n}^{\prime} L / 2}+\zeta_{n} \frac{\sin \zeta_{n} s}{\cos \zeta_{n} L / 2}\right), \quad n \text { odd, } \\
& \psi_{n}(s)=\sqrt{\frac{c_{n}}{L}}\left(\zeta_{n}^{\prime} \frac{\cosh \zeta_{n}^{\prime} s}{\sinh \zeta_{n}^{\prime} L / 2}-\zeta_{n} \frac{\cos \zeta_{n} s}{\sin \zeta_{n} L / 2}\right), \quad n \text { even, }
\end{aligned}
$$

with

$$
\zeta_{n}^{\prime 2}-\zeta_{n}^{2}=\frac{2 \nu}{\epsilon}, \quad \xi_{0}=0, \quad \xi_{n}=k_{B} T\left(\epsilon \zeta_{n}^{4}+2 \nu \zeta_{n}^{2}\right)
$$

The above eigenfunctions are different form those of the Harris-Hearst model. ${ }^{28,68}$ The constants $c_{n}$ follow from the normalization condition and the wave numbers $\zeta_{n}$ and $\zeta_{n}^{\prime}$ are determined by the boundary conditions (2) and (3). ${ }^{56,66,67,71}$ The amplitudes $\boldsymbol{\chi}_{n}(t)$ obey the equations

$$
\frac{d}{d t} \boldsymbol{\chi}_{n}(t)=\sum_{m=0}^{\infty} H_{n m}\left(-\frac{3 \pi \eta}{\tau_{m}} \boldsymbol{\chi}_{m}(t)+\boldsymbol{\Gamma}_{m}(t)\right)+\mathbf{K} \boldsymbol{\chi}_{n},
$$

where $H_{n m}=Q_{n m}+\delta_{n m} /(3 \pi \eta)$,

$$
\begin{gathered}
Q_{n m}=\iint_{-L / 2}^{L / 2} \psi_{n}\left(s^{\prime}\right) Q\left(s-s^{\prime}\right) \psi_{n}(s) d s d s^{\prime}, \\
\left\langle\Gamma_{n \alpha}(t) \Gamma_{m \beta}\left(t^{\prime}\right)\right\rangle=2 k_{B} T \delta_{\alpha \beta} \delta\left(t-t^{\prime}\right) / H_{n m},
\end{gathered}
$$

and $\alpha, \beta \in\{x, y, z\}$. The free draining relaxation times $\tau_{n}$ $=3 \pi \eta / \xi_{n}$ depend on shear rate via $\nu$.

A full solution of Eq. (11) requires diagonalization of the matrix $\mathbf{Q}^{58,66,67}$ However, the diagonal elements typically dominate for the whole range of flexibility parameters $p L$ and are close to the full eigenvalues of $\mathbf{Q}^{25,66,67,78}$ Therefore, only the diagonal elements $Q_{n n}$ will be considered in the following. A numerical solution taking into account a subblock of the slowest relaxation modes is presented in Refs. 58 and 59 for semiflexible polymers. A comparison with mean square displacement data of DNA molecules in solution obtained by fluorescence correlation spectroscopy measurements ${ }^{57}$ yields excellent agreement ${ }^{58}$ and shows that the analytical solution with the diagonal elements only overestimates the mean square displacement only slightly for time scales longer than the bending relaxation time. ${ }^{59}$ This difference can be corrected by taking into account a scale factor for the hydrodynamic interactions, which yields slightly larger relaxation times and provides an excellent agreement between theory and experiments on DNA. ${ }^{57}$

The mode decoupled, but spatial coupled Eq. (11) can easily be solved and amplitude correlation functions be determined, which enter in calculations of expectation values. ${ }^{29,56,66,67,71}$ We find $\left\langle\chi_{n \alpha}(t) \chi_{m \beta}\left(t^{\prime}\right)\right\rangle$ $=\delta_{n m}\left\langle\chi_{n \alpha}(t) \chi_{n \beta}\left(t^{\prime}\right)\right\rangle$ with

$$
\left\langle\chi_{n x}(t) \chi_{n x}(0)\right\rangle=\frac{k_{B} T \tau_{n}}{3 \pi \eta} e^{-|t| \mid \widetilde{\tau}_{n}}\left(1+\frac{\dot{\gamma}^{2} \widetilde{\tau}_{n}}{2}\left(|t|+\widetilde{\tau}_{n}\right)\right),
$$

$$
\begin{aligned}
& \left\langle\chi_{n y}(t) \chi_{n y}(0)\right\rangle=\frac{k_{B} T \tau_{n}}{3 \pi \eta} e^{-|t| \mid \tau_{n}}, \\
& \left\langle\chi_{n x}(t) \chi_{n y}(0)\right\rangle=\frac{k_{B} T \tau_{n} \dot{\gamma}}{3 \pi \eta} e^{-|t| \mid \widetilde{\tau}_{n}}\left(t \Theta(t)+\frac{\widetilde{\tau}_{n}}{2}\right), \\
& \left\langle\chi_{n y}(t) \chi_{n x}(0)\right\rangle=\frac{k_{B} T \tau_{n} \dot{\gamma}}{3 \pi \eta} e^{-|t| \mid \widetilde{\tau}_{n}}\left(t \Theta(-t)+\frac{\widetilde{\tau}_{n}}{2}\right),
\end{aligned}
$$

and $\left\langle\chi_{n z}(t) \chi_{n z}(0)\right\rangle=\left\langle\chi_{n y}(t) \chi_{n y}(0)\right\rangle$. All other correlations are zero. The relaxation times $\widetilde{\tau}_{n}$ are given by

$$
\tilde{\tau}_{n}=\frac{\tau_{n}}{1+3 \pi \eta Q_{n n}},
$$

and depend on hydrodynamic interactions. As expressed by these relations, shear flow induces correlations in time and the history of time evolution of the polymer is important for its conformations and dynamical behavior.

\section{B. Lagrangian multiplier}

The Lagrangian multiplier $\nu=3 p \mu / 2$ follows from Eq. (4), where $\mu=\mu(\dot{\gamma})$ captures the shear rate dependence and $\mu(0)=1$. By inserting the eigenfunction expansion (7) one obtains from Eq. (4)

$$
\sum_{n=1}^{\infty}\left\langle\boldsymbol{\chi}_{n}(0)^{2}\right\rangle \phi_{n}=1,
$$

with

$$
\left\langle\boldsymbol{\chi}_{n}(0)^{2}\right\rangle=\frac{k_{B} T}{\pi \eta} \tau_{n}+\frac{k_{B} T \dot{\gamma}^{2}}{6 \pi \eta} \tau_{n} \widetilde{\tau}_{n}^{2},
$$

and $\phi_{n}=\int\left(\partial \psi_{n}(s) / \partial s\right)^{2} d s / L$. A numerical solution of the equation yields $\mu(\dot{\gamma})$.

The sum in Eq. (16) over the first term on the right hand side of Eq. (17) can be evaluated analytically with the help of the partition function ${ }^{65}$ 


$$
\begin{aligned}
& \frac{k_{B} T}{\pi \eta L} \sum_{n=1}^{\infty} \tau_{n} \phi_{n} \\
& \quad=\frac{\left[\frac{1}{2 p L}\left(1-\frac{1}{\mu}\right)+2\right] \tanh (2 p L \sqrt{\mu})+\frac{1}{\sqrt{\mu}}+\sqrt{\mu} p_{p L \rightarrow \infty}}{(1+\mu) \tanh (2 p L \sqrt{\mu})+2 \sqrt{\mu}} \rightarrow \frac{1}{\sqrt{\mu}} .
\end{aligned}
$$

This expression is governed by the bending modes. Without bending modes, the sum would not converge.

To arrive at an analytical solution, we first consider the limit of a flexible polymer, i.e., $p L \gg 1$, for which

$$
\psi_{n}(s)= \begin{cases}\sqrt{\frac{2}{L}} \sin \left(\zeta_{n} s\right), & n \text { odd } \\ \sqrt{\frac{2}{L}} \cos \left(\zeta_{n} s\right), & n \text { even }\end{cases}
$$

and $\zeta_{n}=n \pi / L$, which yields $\phi_{n}=\zeta_{n}^{2} / L$. Then, the sum over the second term of Eq. (17) results in

$$
\sum_{n=1}^{\infty} \frac{k_{B} T \dot{\gamma}^{2}}{6 \pi \eta L} \tau_{n} \widetilde{\tau}_{n}^{2} \zeta_{n}^{2 L, \mu \gg 1} \rightarrow \frac{\mathrm{Wi}^{2} \pi^{4}}{540 p L \mu^{3}}\left(1+\frac{\pi^{2}}{4 p^{2} L^{2}}\right)^{2},
$$

where $\quad \zeta_{n}^{2} \tau_{n} / L \approx \pi \eta /\left(k_{B} T p L \mu\right) \quad$ and $\quad \widetilde{\tau}_{n} / \widetilde{\tau}_{1}^{0}$ $\approx\left[1+\pi^{2} /\left(4 p^{2} L^{2}\right)\right] /\left(\mu n^{2}\right)$ has been used, i.e., for the latter hydrodynamic interactions are neglected. The Weissenberg number $\mathrm{Wi}=\dot{\gamma} \widetilde{\tau}_{1}^{0}$ is introduce, with $\widetilde{\tau}_{1}^{0}$ the longest relaxation time at equilibrium. Then, $\mu$ follows from

$$
\mu^{3}-\mu^{5 / 2}-\frac{\pi^{4} \mathrm{Wi}^{2}}{540 p L}\left(1+\frac{\pi^{2}}{4 p^{2} L^{2}}\right)^{2}=0 .
$$

For $\mathrm{Wi} \gg 1$, the equation yields

$$
\mu=\mathrm{Wi}^{2 / 3}\left(\frac{\pi^{4}}{540 p L}\right)^{1 / 3} \text {. }
$$

$\mu$ depends on the product $p L$ and is essentially independent of hydrodynamic interactions within the preaveraging approximation. The latter is a consequence of the fact that (i) the sum over modes is dominated by the first mode due to the fast decay of other summands. The ratio between the sums of Rouse and Zimm modes is $\sum_{n=1}^{\infty} 1 / n^{4} / \sum_{n=1}^{\infty} 1 / n^{3}$ $=0.9$, i.e., the error is at the worst $10 \%$, but Zimm modes are obtained for $p L \gtrsim 10^{4}$ only, a ratio much larger than typical values for DNA molecules. (ii) The dependence on hydrodynamic interactions is adsorbed in the Weissenberg number.

Equation (21) suggests that for polymers with $p L \gtrsim 10$ a length and persistence length independent behavior is obtained, when (measured) quantities are presented in terms of $\mathrm{Wi} / \sqrt{p L}$. The chain and persistence length dependents enters via the relaxation times $\tau_{n}$ and $\phi_{n}$; both are equilibrium properties. It has to be kept in mind that all polymer properties depend on $\mu$ and hence on $p L$.

In the limit of a semiflexible chain, i.e., $p L<1$, and for $\mu \rightarrow \infty, \tau_{1}^{0}=\pi \eta L^{3} /\left(12 k_{B} T\right)$ and $\tau_{n} / \tau_{1}^{0} \approx 12 /\left(\mu p L n^{2}\right)$, because the contribution of the bending modes vanishes in the limit $\mu \rightarrow \infty$ as will be discussed in Sec III A. Hydrodynamic interactions are even less important for semiflexible polymers

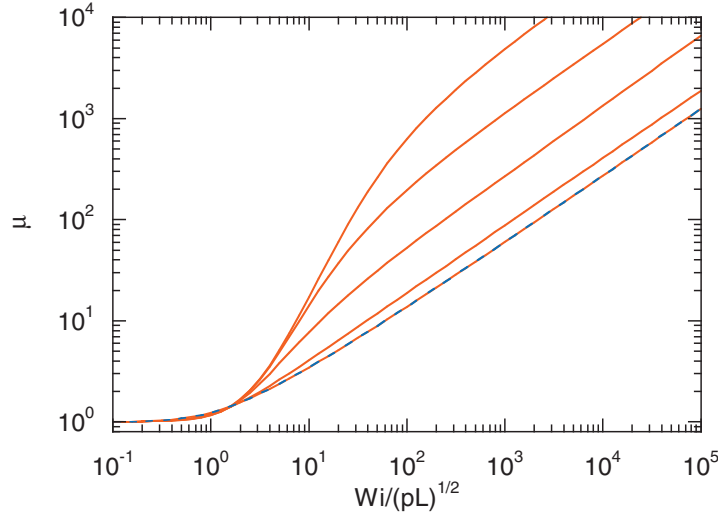

FIG. 1. Lagrangian multipliers $\mu$ as function of the Weissenberg number $\mathrm{Wi}=\dot{\gamma} \tilde{\tau}_{1}^{0}$ for $p L=10^{-3}, 10^{-2}, 10^{-1}, 10^{0}$, and $10^{1}$ (left to right). The lines for larger $p L$ are indistinguishable from that for $p L=10$. The blue dashed line indicates the analytical solution Eq. (22).

than for flexible ones, because the integrals of Eq. (12) yield a logarithmic correction in $n$ only. ${ }^{67,71}$ Therefore, $\mu$ is given by

$$
\mu=\left(\frac{4}{15}\right)^{1 / 3} \frac{\mathrm{Wi}^{2 / 3}}{p L},
$$

in this limit. Hence, a different chain length dependence is obtained for flexible and semiflexible polymers, if the Weissenberg number is defined via the longest relaxation time at equilibrium. The theoretical result compares well with the data of Fig. 1 in the respective limit.

In the limit $\mathrm{Wi} \rightarrow 0, \mu$ is given by

$$
\mu= \begin{cases}1+\mathrm{Wi}^{2} /(6 p L), & p L \ll 1 \\ 1+\mathrm{Wi}^{2} \pi^{4} /(270 p L), & p L \gg 1 .\end{cases}
$$

The full numerical solution is presented in Fig. 1 for various values of $p L$. As predicted by the analytical solution (21), a limiting curve is assumed for $p L \gg 1$. In contrast, for $p L<1$ a strong dependence is obtained on persistence length. However, in the limit $\mathrm{Wi} \rightarrow \infty, \mu$ exhibits the dependence $\mu \sim \mathrm{Wi}^{2 / 3}$ even for semiflexible and rather stiff polymers. This is a result of the $\mu$ dependence of the relaxation times as will be discussed Sec. III A. Introducing the Weissenberg number $\mathrm{Wi}_{z}=\dot{\gamma} \tau_{z}$, where $\tau_{z}$ is the Zimm relaxation, i.e., the longest relaxation time without bending modes, Fig. 2 shows that the curves for the various $p L$ values approach the limiting behavior given by Eq. (22).

I like to emphasize that the dependence of $\mu$ on persistence length is not an artifact by the constraint Eq. (4). The solution of a discrete model with bond constraints for every individual bond yields qualitatively and quantitatively the same dependence for the average over all bonds in the continuum limit.

\section{RESULTS}

\section{A. Relaxation times}

The relaxation times $\tau_{n}$ [Eq. (15)] depend on the shear rate via the Lagrangian multiplier $\mu$ 


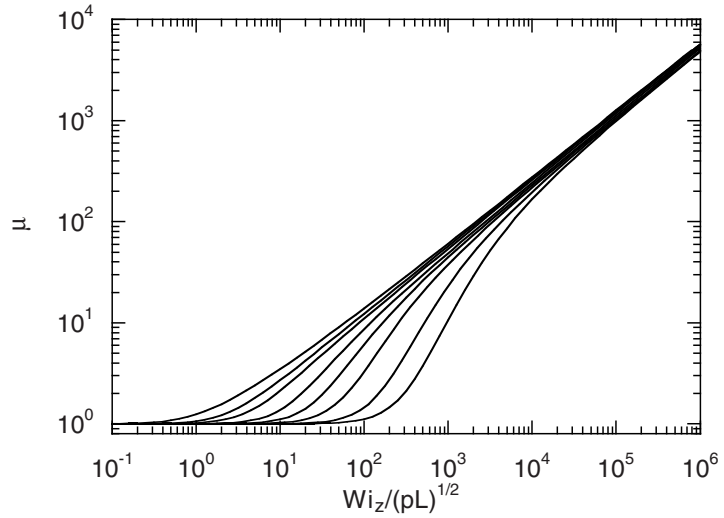

FIG. 2. Lagrangian multipliers $\mu$ as function of the Weissenberg number $\mathrm{Wi}_{z}=\dot{\gamma} \tau_{z}$ for $\mathrm{pL}=10^{4}, 10^{0}, 5 \times 10^{-1}, 2 \times 10^{-1}, 10^{-1}, 5 \times 10^{-2}$, and $2 \times 10^{-2}$, $10^{-2}$ (left to right). $\tau_{z}$ is the longest relaxation time of a polymer without bending modes. The thickness of the polymer is $d / L=10^{-4}$.

$$
\tau_{n}=\frac{\pi \eta}{\mu p k_{B} T \zeta_{n}^{2}}\left[1+\frac{\zeta_{n}^{2}}{4 p^{2} \mu}\right]^{-1}
$$

The bending contribution $\sim \zeta_{n}^{4}$ vanishes in the limit $p L \sqrt{\mu}$ $\rightarrow \infty$ for any $p L$ and the relaxation times become equal to those of a flexible polymer, i.e., under the influence of shear, a polymer behaves more and more like a flexible polymer with increasing Weissenberg number.

This is illustrated in Figs. 3 and 4. In Fig. 3, the relaxation times exhibit a crossover from stretching $(n<100)$ to bending $(n>100)$ mode dominated behavior for $\mu=1 .^{67,70}$ The crossover shifts to large mode numbers with increasing shear rate and the flexible mode regime increases. At the same time, the relaxation times decrease because $\tau_{n} \sim \mu^{-1}$ and the polymer dynamics becomes faster. Since $p L \gg 1$, hydrodynamic interactions contribute to the mode dependence of $\widetilde{\tau}_{n}$. However, the relaxation times do not exhibit the dependence $\widetilde{\tau}_{n} \sim n^{-3 / 2}$ of the Zimm times. ${ }^{5,77}$ This asymptotic behavior is only obtained for $p L \gtrsim 10^{4}$.

Figure 4 displays the dependence of the longest relaxation time on persistence length and Weissenberg number. For $p L \rightarrow 0$-more precisely for $\left.(p L)^{2} \mu \ll 1\right)$ — the first mode and relaxation time are given by

$$
\zeta_{1} L=[48 p L(1+p L \mu)]^{1 / 4}
$$

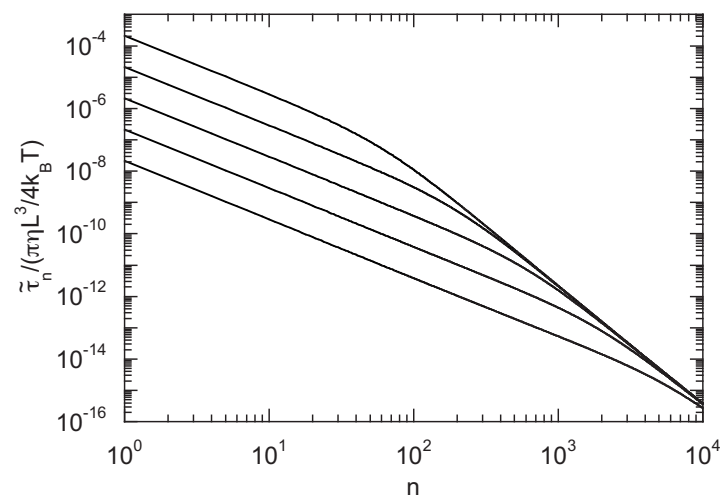

FIG. 3. Relaxation times $\widetilde{\tau}_{n}$ as function of mode number for $\mu$ $=1,10,10^{2}, 10^{3}, 10^{4}$ (top to bottom), $p L=10^{2}$, and $d / L=10^{-4}$.

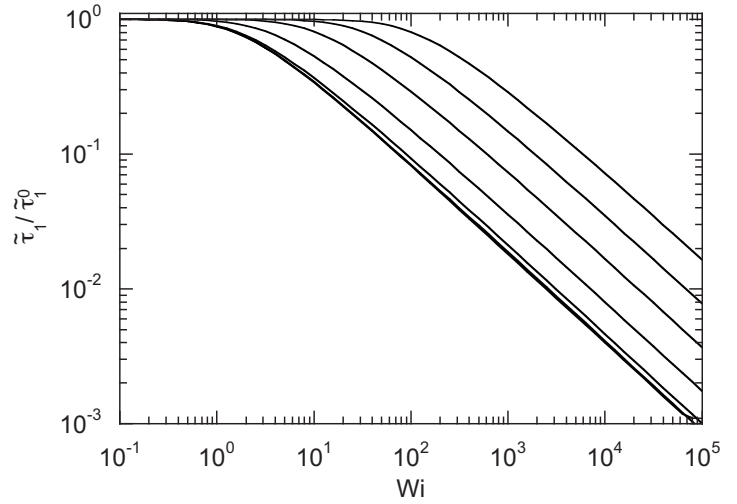

FIG. 4. Relative longest relaxation times $\widetilde{\tau}_{1} / \widetilde{\tau}_{1}^{0}$ as function of the Weissenberg number for $p L=10^{-2}, 10^{-1}, 10^{0}, 10^{1}, 10^{2}, 10^{3}, 10^{4}$ (left to right) and $d / L=10^{-4}$.

$$
\tau_{1}=\frac{\pi \eta L^{3}}{12 k_{B} T(1+p L \mu)}=\tau_{1}^{0} \frac{1+p L}{1+p L \mu} .
$$

The latter term is essentially independent of $p L$, because (1 $+p L \mu) /(1+p L) \approx 1+p L(\mu-1) \approx 1+\mathrm{Wi}^{2} / 6$ for $\mathrm{Wi} \ll 1$ and $\approx(4 / 15)^{1 / 3} \mathrm{Wi}^{2 / 3}$ for $\mathrm{Wi} \gg 1$. A comparison with the numerically calculated relaxation times shows that the ratio is very well approximated by $\widetilde{\tau}_{1} / \widetilde{\tau}_{1}^{0}=(1+p L) /(1+p L \mu)$ for $p L \ll 1$. This ratio depends only very weakly on $p L$. Correspondingly, the curves become indistinguishable for $p L<10^{-1}$ in Fig. 4.

The bending contribution to the relaxation times vanishes in the limit $p L \sqrt{\mu} \gg 1$ independent of $p L$, and the polymer behavior is governed by the flexible modes $\tau_{n}$ even for $p L \ll 1$. However, the hydrodynamic contribution $1 /(1$ $\left.+3 \pi \eta Q_{n n}\right)$ to the relaxation times is always determined by the actual value of $p L$, and a logarithmic correction in $n$ is obtained for $p L \ll 1 .^{67,71}$

\section{B. Conformations}

The polymer conformations under flow are characterized by the mean square end-to-end distance tensor $\mathbf{E}$, with the components $\quad E_{\alpha \beta}=\left\langle\left(r_{\alpha}(L / 2)-r_{\alpha}(-L / 2)\right)\left(r_{\beta}(L / 2)-r_{\beta}(\right.\right.$ $-L / 2))\rangle$, and the radius of gyration tensor $\mathbf{G}$ with the components $G_{\alpha \beta}=\int_{-L / 2}^{L / 2}\left\langle\left(r_{\alpha}(s)-r_{\alpha, c m}\right)\left(r_{\beta}(s)-r_{\beta, c m}\right)\right\rangle d s / L$, where $\boldsymbol{r}_{c m}$ denotes the center-of-mass position of the polymer. ${ }^{63}$ At equilibrium, the polymer is isotropic in average and the mean square end-to-end distance and radius of gyration are given by ${ }^{56,63}$

$$
\begin{aligned}
& \left\langle\boldsymbol{r}_{E}^{2}\right\rangle=\sum_{\alpha=1}^{3} E_{\alpha \alpha}=\frac{L}{p}-\frac{1}{2 p^{2}}\left[1-e^{-2 p L}\right], \\
& \left\langle\boldsymbol{r}_{G}^{2}\right\rangle=\sum_{\alpha=1}^{3} G_{\alpha \alpha}=\frac{L}{6 p}-\frac{1}{4 p^{2}}+\frac{1}{4 p^{3} L}-\frac{1}{8 p^{4} L^{2}}\left[1-e^{-2 p L}\right] .
\end{aligned}
$$

With the eigenfunction expansion (7) and the correlation functions (14), the tensor elements read 


$$
\begin{aligned}
& E_{\alpha \alpha}=E_{\alpha \alpha}^{(1)}+\frac{2 k_{B} T \mathrm{Wi}^{2}}{3 \pi \eta L} \sum_{n, o d d}^{\infty} \psi_{n}^{2}\left(\frac{L}{2}\right) \tau_{n}\left(\frac{\tau_{n}}{\tau_{1}^{\theta}}\right)^{2} \delta_{\alpha x}, \\
& G_{\alpha \alpha}=G_{\alpha \alpha}^{(1)}+\frac{k_{B} T \mathrm{Wi}^{2}}{6 \pi \eta L} \sum_{n=1}^{\infty} \tau_{n}\left(\frac{\widetilde{\tau}_{n}}{\widetilde{\tau}_{1}^{0}}\right)^{2} \delta_{\alpha x},
\end{aligned}
$$

where

$$
\begin{aligned}
E_{\alpha \alpha}^{(1)} & =\frac{L}{3 p \mu}-\frac{1}{3 p^{2} \mu^{3 / 2}(\sqrt{\mu}+\operatorname{coth}(p L \sqrt{\mu}))}, \\
G_{\alpha \alpha}^{(1)} & =\frac{k_{B} T}{3 \pi \eta L} \sum_{n=1}^{\infty} \tau_{n} .
\end{aligned}
$$

For $p L \gg 1$, the expressions reduce to

$$
\begin{gathered}
E_{\alpha \alpha}=\frac{\left\langle\boldsymbol{r}_{E}^{2}\right\rangle}{3 \mu}+\frac{\pi^{4} L^{2} \mathrm{Wi}^{2}}{720 p L \mu^{3}} \delta_{\alpha x}, \\
G_{\alpha \alpha}=\frac{\left\langle\boldsymbol{r}_{G}^{2}\right\rangle}{3 \mu}+\frac{\pi^{4} L^{2} \mathrm{Wi}^{2}}{5670 p L \mu^{3}} \delta_{\alpha x},
\end{gathered}
$$

and for $p L \ll 1$, we find

$$
\begin{gathered}
E_{\alpha \alpha} \\
= \begin{cases}\frac{L^{2}}{3(1+p L \mu)}\left(1+\frac{\mathrm{Wi}^{2}}{2}\left(\frac{1+p L}{1+p L \mu}\right)^{2} \delta_{\alpha x}\right), & p L \sqrt{\mu}<1 \\
\frac{L^{2}}{3 p L \mu}\left(1+\frac{3 \mathrm{Wi}^{2}}{5(p L \mu)^{2}} \delta_{\alpha x}\right), & p L \sqrt{\mu} \gg 1,\end{cases} \\
G_{\alpha \alpha}= \begin{cases}E_{\alpha \alpha} / 12, & p L \sqrt{\mu}<1 \\
\frac{L^{2}}{18 p L \mu}\left(1+\frac{144 \mathrm{Wi}^{2}}{315(p L \mu)^{2}} \delta_{\alpha x}\right), & p L \sqrt{\mu} \gg 1,\end{cases}
\end{gathered}
$$

if only the first mode is taken into account for $p L \sqrt{\mu} \ll 1$, and for $p L \sqrt{\mu} \gg 1$ the relaxation times $\tau_{n}$ of Eq. (25) and $\tau_{1}^{0}$ of Eq. (27) are used. As discussed before, hydrodynamic interactions have been neglected. They yield an even smaller contribution as in the calculation of $\mu$.

Figure 5 shows mean square end-to-end distances and radii of gyration for various persistence lengths. The polymers extend along the flow direction and saturate at large Weissenberg numbers due the finite chain extensibility. For $p L>1$, a monotonous increase is obtained, whereas for $p L$ $<1, E_{x x}$ passes through the maximum $E_{x x} / E_{x x}^{0} \approx 3$, where $E_{x x}^{0}=\left\langle\boldsymbol{r}_{E}^{2}\right\rangle / 3$ and approaches a plateau value at large Wi. Correspondingly, $G_{x x}$ seems to assume the value $G_{x x} / G_{x x}^{0} \approx 3$, where $G_{x x}^{0}=\left\langle\boldsymbol{r}_{G}^{2}\right\rangle / 3$, before it finally approaches a larger limiting value. The nonmonotonous behavior of the stiff polymers can be explained as follows: Since they resemble rodlike objects, the shear flow aligns them in the flow direction. This implies a maximal change of extension by a factor three, which corresponds to the observed ratio. For even larger Wi the influence of bending modes decreases, i.e., the polymers become more flexible. Hence, the mean square end-to-end distances decrease and the radii of gyration in-
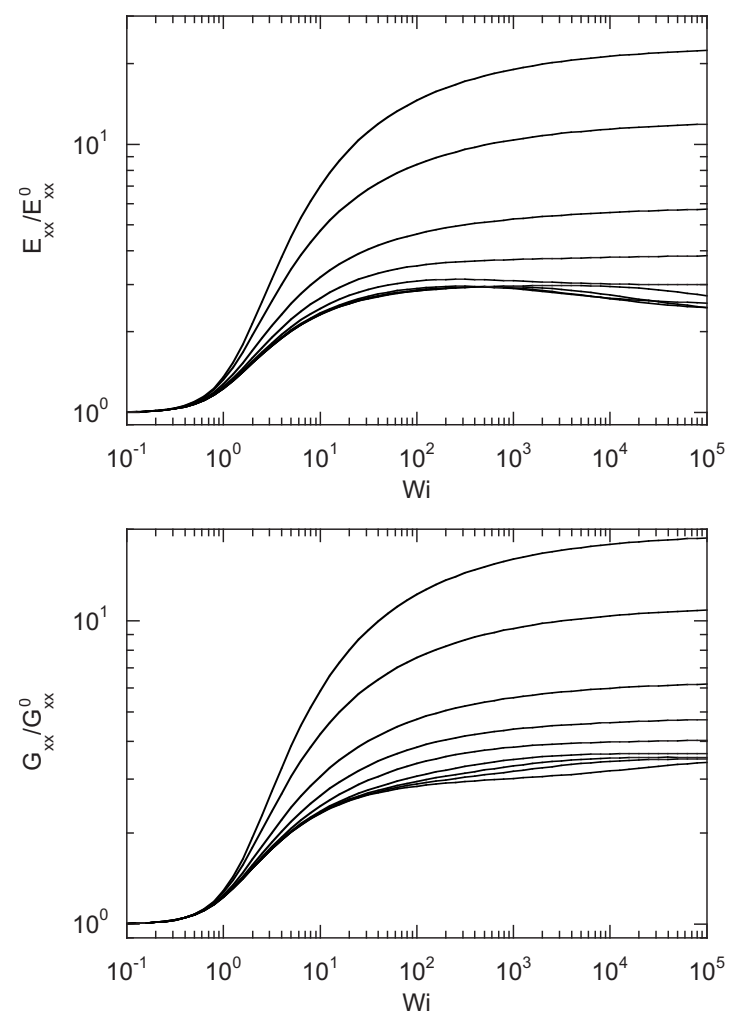

FIG. 5. Relative mean square end-to-end distance (top) and radius of gyration (bottom) along the flow direction for $p L=10,5,2,1,0.5,0.2,0.1,5$ $\times 10^{-2}, 10^{-2}$ (top to bottom) and $d / L=10^{-4}$.

crease. The crossover to the flexible behavior depends on persistence length and appears at large Wi for stiffer polymers. Therefore, the maximum in $E_{x x}$ shifts to larger Wi with decreasing $p L$.

The polymers shrink transverse to the flow direction, where the model yields the same behavior in the gradient and vorticity direction, although simulations show that the two directions are inequivalent due to excluded volume interactions. $^{53,79}$ The relative end-to-end distance obeys $E_{y y} / E_{y y}^{0}=(1+p L) /(1+p L \mu)$ for all $\mathrm{Wi}$, as is obvious from Eqs. (31)-(33). The radius of gyration follows the dependence $G_{y y} / G_{y y}^{0}=(1+p L) /(1+p L \mu)$ for $p L \sqrt{\mu}<1$ and $G_{y y} / G_{y y}^{0}=2 /(p L \mu)$ for $p L \sqrt{\mu} \gg 1$, respectively. The latter ratio expresses the difference between the radius of gyration of a flexible and a rodlike polymer. Hence, this component of the end-to-end distance and the radius of gyration of semiflexible and stiff polymers exhibits the limiting behavior $G_{y y} / G_{y y}^{0} \sim \mathrm{Wi}^{-2 / 3}$ for $p L \sqrt{\mu} \gg 1$. Experiments and simulations yield a similar value in the corresponding limit. ${ }^{9,42}$

In the limit $p L \ll 1$, the off-diagonal component $G_{x y}$ is well approximated by

$$
G_{x y}= \begin{cases}\frac{\mathrm{Wi}(1+p L)^{2} L^{2}}{72(1+p L \mu)^{2}}, & p L \sqrt{\mu}<1 \\ \frac{\mathrm{Wi}(1+p L) L^{2}}{45(p L \mu)^{2}}, & p L \sqrt{\mu} \gg 1 .\end{cases}
$$

Since $p L \mu$ is independent of the persistence length in that limit, a universal curve is obtained. For a flexible polymer 


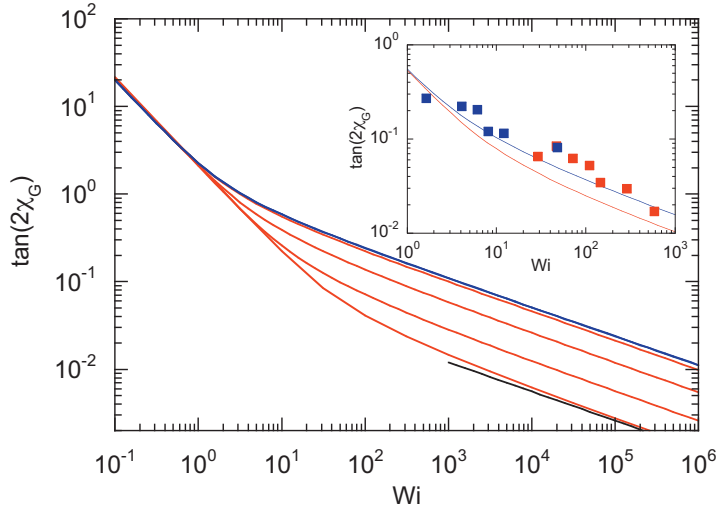

FIG. 6. Alignment angle as function of Weissenberg number for the stiffnesses $p L=10^{-2}, 10^{-1}, 10^{0}, 10^{1}, 10^{2}, 10^{3}$ (right to left) and $d / L=10^{-4}$. The black line indicates the asymptotic limit for $\mathrm{Wi} \rightarrow \infty$ according to the expression (38), i.e., it is proportional to $\mathrm{Wi}^{-1 / 3}$. Inset: Comparison of theoretical results with measurements on DNA molecules of length $L=48.5 \mathrm{kbp}$ $(\lambda$-DNA, $p L=150)$ (blue) and $L=185 \mathrm{kbp}(p L=570)$ (red). The experimental values (squares) are taken from Ref. 9.

$$
G_{x y}=\frac{\pi^{2} \mathrm{Wi} L^{2}}{540 p L \mu^{2}} .
$$

Hence, the off-diagonal element increases linearly with the Weissenberg number for $\mu \ll 1$ and decrease as $\mathrm{Wi}^{-1 / 3}$ for $\mathrm{Wi} \rightarrow \infty$.

The theoretical model yields maximum values for $E_{x x}^{m}$ and $G_{x x}^{m}$ in the limit $\mathrm{Wi} \rightarrow \infty$ that seem to be larger than those obtained experimentally. This is due to contour lengths fluctuations of the Gaussian model. However, the deviation, e.g., $1-E_{x x} / E_{x x}^{m}$ from the limiting value is proportional to $\mathrm{Wi}^{-1 / 3}$ for both, $E_{x x}$ and $G_{x x}$. This is in agreement with the scaling considerations and simulations of Ref. 11.

\section{Alignment}

Aside from deformation, polymers exhibit a preferred alignment in shear flow, as has been shown experimentally, in computer simulations, and theoretical calculations. The alignment can be characterized by the angle $\chi_{G}$, which is the angle between the major axis of the gyration tensor and the flow direction. It is obtained from $\mathbf{G}$ as

$$
\tan \left(2 \chi_{G}\right)=\frac{2 G_{x y}}{G_{x x}-G_{y y}} .
$$

With the expressions (32) and (34), we find for $p L \gg 1$

$$
\tan \left(2 \chi_{G}\right)=\frac{21 \mu}{\pi^{2} \mathrm{Wi}},
$$

and for $p L \ll 1$

$$
\tan \left(2 \chi_{G}\right)= \begin{cases}2(1+p L \mu) / \mathrm{Wi}, & p L \sqrt{\mu}<1 \\ 7 p L \mu /(4 \mathrm{Wi}), & p L \sqrt{\mu} \gg 1 .\end{cases}
$$

Hence, in any case $\tan \left(2 \chi_{G}\right) \sim \mathrm{Wi}^{-1 / 3}$ in the limit $\mathrm{Wi} \rightarrow \infty$.

Figure 6 displays $\tan \left(2 \chi_{G}\right)$ for various persistence lengths. There is a clear length dependence for flexible polymers, whereas a limiting curve is assumed for stiff polymers. In the inset, experimental data are compared with theoretical predictions. A detailed discussion is provided in Sec. V.

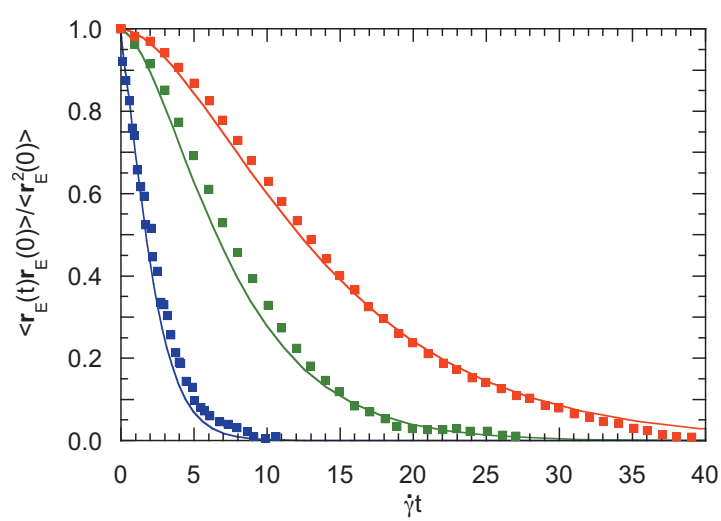

FIG. 7. Theoretical end-to-end vector correlation functions (solid lines) for the Weissenberg numbers Wi=5.2 (blue), 25 (green), and 80 (red) for $p L$ $=150$ and $d / L=10^{-4}$. The squares are experimental results of the extension autocorrelation function for $\lambda$-DNA extracted from Ref. 1 for $\mathrm{Wi}=1.3$ (blue), 6.3 (green), and 19 (red).

\section{End-to-end vector relaxation}

The nonequilibrium dynamics of a polymer depends on shear rate. As an example, the end-to-end vector relaxation behavior is addressed. The end-to-end vector correlation function reads

$$
\begin{aligned}
\left\langle\boldsymbol{r}_{E}(t) \boldsymbol{r}_{E}(0)\right\rangle= & \sum_{n, \text { odd }} 4 \psi_{n}^{2}(L / 2)\left\langle\boldsymbol{\chi}_{n}(t) \boldsymbol{\chi}_{n}(0)\right\rangle \\
= & \frac{4 k_{B} T}{\pi \eta} \sum_{n, o d d} \psi_{n}^{2}(L / 2) \tau_{n}\left(1+\frac{\mathrm{Wi}^{2}}{6}\left(\frac{\widetilde{\tau}_{n}}{\widetilde{\tau}_{1}^{0}}\right)^{2}\right. \\
& \left.+\frac{\mathrm{Wi}}{6} \frac{\dot{\gamma} t \widetilde{\tau}_{n}}{\widetilde{\tau}_{1}^{0}}\right) \exp \left(-\frac{\dot{\gamma} t \tau_{1}^{0}}{\mathrm{Wi} \widetilde{\tau}_{n}}\right),
\end{aligned}
$$

with the correlation functions (14). Here, the combination $\dot{\gamma} t$ is used as dimensionless quantity. Since $\mathrm{Wi}=\dot{\gamma} \widetilde{\tau}_{1}^{0}$, $\dot{\gamma} \tilde{\tau} \tau_{1}^{0} /\left(\mathrm{Wi} \widetilde{\tau}_{n}\right)=t / \widetilde{\tau}_{n}$. For every mode, $\left\langle\boldsymbol{r}_{E}(t) \boldsymbol{r}_{E}(0)\right\rangle$ includes a purely exponentially decaying term and a term linear in time multiplied by the same exponential function. Hence, the correlation function decays in a nonexponential manner for sufficiently large Weissenberg numbers. This is reflected in Fig. 7 , where theoretical results are presented for $\lambda$-DNA-like polymers. At short times, the nonexponential decay is clearly visible.

To obtain an analytical approximation of the full correlation function, only the first mode in Eq. (40) is taken into account, which yields for the normalized correlation function $^{20,28}$

$$
\frac{\left\langle\boldsymbol{r}_{E}(t) \boldsymbol{r}_{E}(0)\right\rangle}{\left\langle\boldsymbol{r}_{E}(0)^{2}\right\rangle}=\left(1+\frac{\mathrm{Wi} \dot{\gamma} t\left(\widetilde{\tau}_{1} / \widetilde{\tau}_{1}^{0}\right) / 6}{1+\mathrm{Wi}^{2}\left(\widetilde{\tau}_{1} / \widetilde{\tau}_{1}^{0}\right)^{2} / 6}\right) \exp \left(-\frac{\dot{\gamma} t \tau_{1}^{0}}{\mathrm{Wi} \widetilde{\tau}_{1}}\right) .
$$

Approximations for the relaxation times are discussed in Sec. II B for large and small $p L$. Specifically, for $p L \gg 1$, Eq. (41) reads 


$$
\frac{\left\langle\boldsymbol{r}_{E}(t) \boldsymbol{r}_{E}(0)\right\rangle}{\left\langle\boldsymbol{r}_{E}(0)^{2}\right\rangle}=\left(1+\frac{\mathrm{Wi} \dot{\gamma} t}{6 \mu\left(1+\mathrm{Wi}^{2} /\left(6 \mu^{2}\right)\right)}\right) \exp \left(-\frac{\dot{\gamma} t \mu}{\mathrm{Wi}}\right) .
$$

This expression depends explicitly and implicitly via the Lagrangian multiplier $\mu$ on the Weissenberg number. The full theoretical curves of Fig. 7 are reasonably well described by this expression.

\section{RHEOLOGY}

\section{A. viscosity}

The polymer contribution $\eta_{p}$ to the viscosity of a (dilute) solution follows from the virial expression of the stress tensor $\sigma_{x y}$ by $\eta_{p}=\sigma_{x y} / \dot{\gamma}$. The stress tensor itself is related to the force density $\boldsymbol{F}$ of Eq. (1) via

$$
\sigma_{x y}=-\frac{1}{V} \int_{-L / 2}^{L / 2}\left\langle F_{x}(s) r_{y}(s)\right\rangle d s,
$$

which yields

$$
\eta_{p}=\frac{k_{B} T}{2 V} \sum_{n=1}^{\infty} \widetilde{\tau}_{n}(\dot{\gamma})
$$

with the eigenfunction expansion (7).

The viscosity is proportional to the relaxation times and depends on the Weissenberg number only via the Lagrangian multiplier $\mu$. Hence, its value, in particular the zero shear rate viscosity $\eta_{p}^{0}$, is more sensitive to the presence of hydrodynamic interactions as compared to the previously discussed expressions, where the ratio $\widetilde{\tau}_{n} / \widetilde{\tau}_{1}^{0}$ enters only.

The relaxation times yield

$$
\eta_{p}= \begin{cases}\frac{\eta_{p}^{0}}{\mu}, & p L \gg 1 \\ \eta_{p}^{0} \frac{1+p L}{1+p L \mu}, & p L \sqrt{\mu}<1,\end{cases}
$$

In the limit of stiff polymers, $\eta_{p}$ is determined by the first relaxation time for $p L \sqrt{\mu} \ll 1$. For $p L \sqrt{\mu} \gg 1$, all modes contribute as for flexible polymers. Here, $\eta_{p} \sim 1 / \mu$, but with a proportionality factor different from $\eta_{p}^{0}$. The factor itself is obtained as sum over $\widetilde{\tau}_{n}=\tau_{n} /\left(1+3 \pi \eta Q_{n n}\right)$, where $\tau_{n}$ is given in Eq. (25) and $Q_{n n}$ (12) is calculated for a semiflexible polymer with the particular $p L$.

In any case, the solution exhibits shear thinning, which is expressed by $\mu$ and is a consequence of the polymer inextensibility. The fact that the decay of the viscosity is governed by $\mu$, i.e., by bond forces, is not surprising, since the model yields a bond contribution to viscosity only.

In the limit $\mathrm{Wi} \gg 1$, the viscosity decreases as $\mathrm{Wi}^{-2 / 3}$. A similar dependence has be found in experiments and simulations. ${ }^{6,7,9,40,42}$ However, experiments and simulations often predicted an exponent closer to $1 / 2 .^{9,41,80}$ This is partially explained by the fact that the crossover is very broad between the zero shear viscosity and the limiting value for $\mathrm{Wi} \rightarrow \infty$. It is easily possible to fit a power-law with an exponent in the range of $1 / 2-2 / 3$ over a decade of Weissenberg numbers in this regime. ${ }^{6,7,9,41-43,50}$

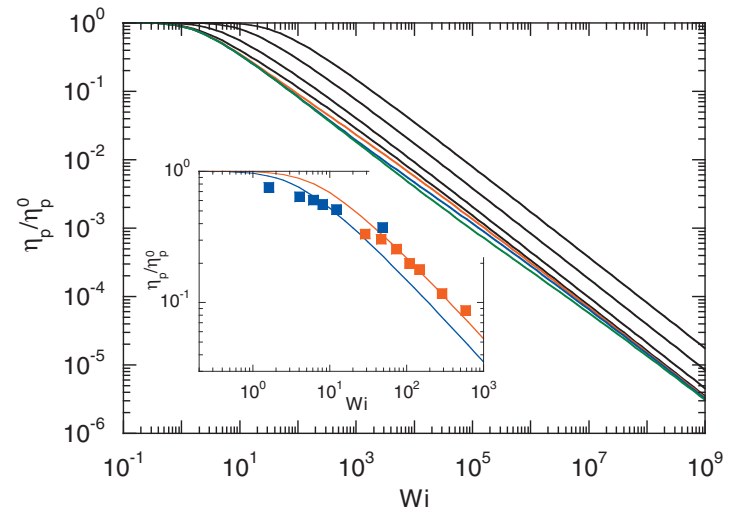

FIG. 8. Polymer contribution to viscosity $\eta_{p} / \eta_{p}^{0}$, where $\eta_{p}^{0}$ is the zero shear value, as function of Weissenberg number for the stiffnesses $p L=10^{-3}$ (green), $10^{-2}$ (blue), $10^{-1}$ (red), $10^{0}, 10^{1}, 10^{2}, 10^{3}$ (left to right) and $d / L$ $=10^{-4}$. Inset: comparison of theoretical results with measurements on DNA molecules of length $L=48.5 \mathrm{kbp}(\lambda$-DNA, $p L=150)$ (blue) and $L$ $=185 \mathrm{kbp}(p L=570)$ (red). The experimental values (squares) are taken from Ref. 9.

For flexible polymers, the viscosity depends on the polymer length via the multiplier $\mu$. Its dependence on molecular weight is discussed in Sec. II B. Such a dependence has also been observed in mesoscale hydrodynamic simulations of polymer solutions. ${ }^{79}$

Stiffness has only a minor influence on the viscosity. In particularly, $\eta_{p}^{0}$ is essentially independent of stiffness for $p L<1$. Due to the change in the nature of the relaxation times from bending modes dominated to stretching modes dominated (flexible) behavior by increasing shear rate, 67,70 there is a change in the slope of $\eta_{p}$ in the vicinity of the Weissenberg numbers where $p L \sqrt{\mu} \approx 1$. As an example, the viscosity for $p L=10^{-2}$ is well described by the power-law $\eta_{p} \sim \mathrm{Wi}^{-0.6}$ for $10^{3}<\mathrm{Wi}<10^{5}$.

This behavior is illustrated in Fig. 8. There is a slight difference in the viscosity for $p L=10^{-1}$ (red) and $p L=10^{-3}$ (green) in the range $10^{2}<\mathrm{Wi}<10^{6}$. A limiting curve is assumed for stiff polymers in the limit $\mathrm{Wi} \rightarrow \infty$.

\section{B. Normal stress difference}

The first normal stress coefficient $\Psi_{1}=\left(\sigma_{x x}-\sigma_{y y}\right) / \dot{\gamma}^{2}$ is given by

$$
\Psi_{1}=\frac{k_{B} T}{2 V} \sum_{n=1}^{\infty} \widetilde{\tau}_{n}^{2}
$$

Since $\Psi_{1}$ is determined by the relaxation times, the considerations of Sec. III apply also, and we find $\Psi_{1} / \Psi_{1}^{0} \sim \mu^{-2}$ $\sim \mathrm{Wi}^{-4 / 3}$ for $\mathrm{Wi} \gg 1$, where $\Psi_{1}^{0}$ is the zero shear normal stress coefficient. Surprisingly, the proportionality $\Psi_{1} / \Psi_{1}^{0}$ $\sim \dot{\gamma}^{-4 / 3}$ is predicted by experiments and a broad spectrum of simulations, ${ }^{9,40-43}$ although the viscosity may display a shear rate dependence different from $\eta_{p} \sim \dot{\gamma}^{-2 / 3}$.

The model does not yield a second normal stress coefficient, in contrast to experimental results. ${ }^{6,7}$ This is related to negligence of excluded volume interactions and fluctuations in hydrodynamic interactions. ${ }^{7}$ As proposed in Refs. 7, 81, and 82 a Gaussian approximation approach yields a nonvanishing and negative $\Psi_{2}$. Computer simulations show that 
$\Psi_{2}$ depends on intramolecular excluded volume as well as hydrodynamic interactions ${ }^{6,7,41}$ and on polymer concentration. $^{79}$

\section{COMPARISON WITH EXPERIMENTS}

Experimental results are presented in Refs. 1, 8, and 9 of conformational, dynamical, and rheological properties of DNA molecules of length $L_{1}=48.5 \mathrm{kbp}\left(\lambda\right.$-DNA) and $L_{2}$ $=185 \mathrm{kbp}$ obtained by fluorescence microscopy. Staining of DNA molecules with YOYO-1 leads to an increase of their contour lengths such that $L_{1}=22 \mu \mathrm{m}^{1,9,83}$ and $L_{2}=84 \mu \mathrm{m} .^{8,9}$ Polymer chain retraction experiments yield the relaxation times $\tau_{1}=28 \mathrm{~s}\left(\lambda\right.$-DNA) and $\tau_{2}=251 \mathrm{~s}$ in a solvent of viscosity $\eta=300 \mathrm{cP}^{8,9}$ The actual value of the persistence length of the stained molecules is not precisely known. The studies of Ref. 84 suggest that $l_{p}$ increases by a factor of 1.32 compared to its native value of $l_{p}=50 \mathrm{~nm}$. Comparing these measurements with our FCS studies of Ref. 57, however, indicates rather a factor of approximately 1.5 than 1.3. Thus, we will use the persistence length $l_{p}$ $=73 \mathrm{~nm}$ in the following, which corresponds to $p L=150$ and 570 , respectively. With these values, the semiflexible chain model yields relaxation times, which are approximately four times larger than the experimental ones. For a quantitative comparison between the results of the theoretical model and the experimental data, four times larger Weissenberg numbers are used, i.e., $\mathrm{Wi}_{\mathrm{th}}=4 \mathrm{Wi}_{\mathrm{ex}}$. The same factor has been used in Ref. 29. In Ref. 29, the factor is motivated by the agreement of the distributions of the alignment angle. Here, the factor seems to be suitable due to the favorable agreement of the viscosities and end-to-end relaxation behavior.

In Ref. 85, the longest relaxation times of $\lambda$-DNA have been determined experimentally for various solvent viscosities. These values are in good agreement with relaxation times calculated by the Zimm model ${ }^{85}$ as well as those obtained by the current semiflexible polymer model; the latter values are approximately $15 \%$ smaller than the Zimm times. For unstained $\lambda$-DNA at the solvent viscosity $\eta=300 \mathrm{cP}$, the theoretical approaches yield relaxation times that are approximately twice as larger as the experimental value $\tau_{1}$ $=28 \mathrm{~s}$ obtained for YOYO-1 stained molecules. The consideration of changes in length and persistence length yields the above mentioned increase in relaxation time by a factor of four compared to $\tau_{1}$. This discrepancy in relaxation times obtained by stress relaxation and chain retraction experiments has already been stressed in Ref. 85. However, it cannot be explained by changes of the persistence length, because by staining we expect an increase of persistence length rather than a decrease, and an increasing $l_{p}$ leads to increasing relaxation times. There seems to be no satisfactory explanation for this discrepancy at the moment. If we, however, assume that the time $\tau_{1}$ underestimates the longest relaxation time of the stained DNA molecule, the actual experimental Weissenberg number would be higher, in agreement with the theoretical assumption. Here, more experimental studies are necessary to resolve the influence of staining on polymer dynamics.
The inset of Fig. 6 displays $\tan \left(2 \chi_{G}\right)$ for the two polymer lengths. The theoretical curves qualitatively capture the decay of the experimental data, but there is a shift to large angles by approximately 1.7 of the experimental data for the longer polymer. However, the experimental data exhibit no polymer length dependence, at least as far as such a conclusion can be drawn from the few data points. Mesoscale hydrodynamic simulations of flexible polymers in dilute solution provide clear evidence for a polymer length dependence of $\chi_{G}{ }^{79}$

The end-to-end vector relaxation behavior is presented in Fig. 7. The experimental data show the decay of the polymer extension correlation function rather than that of the end-to-end vector. Considering this difference, the theoretical model captures the decay of the correlation function well for all three Weissenberg numbers. I would like to point out that the curves of Fig. 7 are also reasonably well described by the analytical expression (42).

Two factors determine the decay of the correlation function, the exponential term $\exp \left(-t / \widetilde{\tau}_{1}\right)$ and the term, which linearly increases with time [cf. Eq. (42)]. A presentation of the time dependence in terms of the variable $t / \widetilde{\tau}_{1}^{0}$, i.e., in terms of the longest relaxation time, eliminates differences in the longest relaxation times between experimental data and theoretical results. However, in Fig. 7 the variable $\dot{\gamma} t$ is used, which implies a dependence of the exponential factor on the Weissenberg number. Hence, for a consistent comparison, the experimental value is taken for Wi. The term, which depends linearly on time is important for $\mathrm{Wi}>1$ and determines the nonexponential shape of the correlation function. The chosen Weissenberg number is evidently suitable to describe the experimental findings.

Finally, the polymer contribution to viscosity is compared in Fig. 8. Aside from the value at the largest Weissenberg number for $\lambda$-DNA, the theoretical curves agree well with the experimental data. The decay at large $\mathrm{Wi}$ is well captured by the theoretical description, the asymptotic dependence $\eta_{p} \sim \mathrm{Wi}^{-2 / 3}$ is reach for even large Weissenberg numbers only.

In summary, the theoretical model provides an adequate description of the nonequilibrium behavior of DNA molecules in shear flow. For certain properties, even a quantitative description is achieved, if the Weissenberg number is adequately chosen.

\section{SUMMARY AND CONCLUSIONS}

In this article the conformational, dynamical, and rheological properties have been determined of a finite extensible Gaussian semiflexible polymer model under shear flow. In particular, the dependence of these properties on polymer stiffness has been addressed.

The model neglects excluded volume interactions and treats hydrodynamic interactions within the preaveraging approximation. Excluded volume interactions are certainly important for flexible polymers at low shear rates and they determine the scaling behavior of such chains with respect to their length. At large shear rates or large stiffnesses, excluded volume interactions are less relevant although even stiff 
polymers behave increasingly flexible with increasing shear rate, as has been shown in Sec. III A. Measurements and simulations of the dynamics of DNA molecules and the comparison with the Gaussian semiflexible polymer model ${ }^{57-59}$ suggest that excluded volume interactions are of minor relevance for DNA lengths $\lesssim 10^{2} \mathrm{~nm}$. The simulations of Ref. 3 and 9 as well as renormalization group studies ${ }^{21}$ lead to the same conclusion. Hence, we can expect the model to reasonably well describe the nonequilibrium properties even to some degree in a quantitative manner. The comparison of various measured quantities with theoretical predictions confirms the expectation.

Hydrodynamic fluctuations seem to be of minor importance for most of the nonequilibrium polymer properties. Hydrodynamic interactions mainly affect the relaxation times, the longest one determines the Weissenberg number. However, hydrodynamic fluctuations are relevant, as expressed by a nonzero second normal stress coefficient, but seem to yield (small) higher order contributions to other quantities only.

A large stiffness does not qualitatively change the shear rate dependent properties of semiflexible polymers. The main difference, compared to a flexible polymer, is the lack of a chain length dependence of these properties. They exhibit rather universal behavior for $p L \ll 1$ and $d / L=$ const. In contrast to flexible polymers, the quantitative values depend on the ratio $d / L$.

A comparison with experimental results suggest that the model provides an adequate qualitative and quantitative description of semiflexible polymer behavior in shear flow. It might be necessary to adjust certain parameters, e.g., the Weissenberg number, to match the theoretical and experimental scales, but otherwise the essence is captured of the underlying physical interactions and mechanisms. This underlines the usefulness of the model for a qualitative and even quantitative understanding of the nonequilibrium properties of flexible and semiflexible polymers.

${ }^{1}$ D. E. Smith, H. P. Babcock, and S. Chu, Science 283, 1724 (1999).

${ }^{2}$ P. LeDuc, C. Haber, G. Boa, and D. Wirtz, Nature (London) 399, 564 (1999).

${ }^{3}$ C. M. Schroeder, R. E. Teixeira, E. S. G. Shaqfeh, and S. Chu, Phys. Rev. Lett. 95, 018301 (2005).

${ }^{4}$ S. Gerashchenko and V. Steinberg, Phys. Rev. Lett. 96, 038304 (2006).

${ }^{5}$ M. Doi and S. F. Edwards, The Theory of Polymer Dynamics (Clarendon, Oxford, 1986).

${ }^{6}$ R. B. Bird, C. F. Curtiss, R. C. Armstrong, and O. Hassager, Dynamics of Polymer Liquids (John Wiley \& Sons, New York, 1987), Vol. 2.

${ }^{7}$ H. C. Öttinger, Stochastic Processes in Polymeric Fluids (Springer, New York, 1996)

${ }^{8}$ R. E. Teixeira, H. P. Babcock, E. S. G. Shaqfeh, and S. Chu, Macromolecules 38, 581 (2005).

${ }^{9}$ C. M. Schroeder, R. E. Teixeira, E. S. G. Shaqfeh, and S. Chu, Macromolecules 38, 1967 (2005).

${ }^{10}$ P. S. Doyle, B. Ladoux, and J.-L. Viovy, Phys. Rev. Lett. 84, 4769 (2000).

${ }^{11}$ B. Ladoux and P. S. Doyle, Europhys. Lett. 52, 511 (2000).

${ }^{12}$ J. A. Y. Johnson, Macromolecules 20, 103 (1987).

${ }^{13}$ W. Bruns and W. Carl, Macromolecules 26, 557 (1993).

${ }^{14}$ W. Carl and W. Bruns, Macromol. Theory Simul. 3, 295 (1994).

${ }^{15}$ W. Carl, Macromol. Theory Simul. 3, 705 (1994).

${ }^{16}$ X. Wang and A. P. Chatterjee, Macromolecules 34, 1118 (2001).

${ }^{17}$ N. J. Woo and E. S. G. Shaqfeh, J. Chem. Phys. 119, 2908 (2003).

${ }^{18}$ M. Chertkov, I. Kolokolov, A. Lebedev, and K. Turitsyn, J. Fluid Mech. 531, 251 (2005).

${ }^{19}$ J. Dubbeldam and F. Redig, J. Stat. Phys. 125, 225 (2006).
${ }^{20}$ A. Dua and B. J. Cherayil, J. Chem. Phys. 112, 8707 (2000).

${ }^{21}$ Y. Rabin and K. Kawasaki, Phys. Rev. Lett. 62, 2281 (1989).

${ }^{22}$ J. R. Prakash, Macromolecules 34, 3396 (2001).

${ }^{23}$ S.-Q. Wang, Phys. Rev. A 40, 2137 (1989).

${ }^{24}$ P. R. Baldwin and E. Helfand, Phys. Rev. A 41, 6772 (1990).

${ }^{25}$ F. Ganazzoli and G. Raffaini, Macromol. Theory Simul. 8, 234 (1999).

${ }^{26}$ A. Subbotin, A. Semenov, E. Manias, G. Hadziioannou, and G. ten Brinke, Macromolecules 28, 3898 (1995).

${ }^{27}$ R. Prabhakar and J. R. Prakash, J. Rheol. 50, 561 (2006).

${ }^{28}$ A. Dua and B. J. Cherayil, J. Chem. Phys. 113, 10776 (2000).

${ }^{29}$ R. G. Winkler, Phys. Rev. Lett. 97, 128301 (2006).

${ }^{30}$ T. Munk, O. Hallatschek, C. H. Wiggins, and E. Frey, Phys. Rev. E 74, 041911 (2006).

${ }^{31}$ K. D. Knudsen, A. Elgsaeter, J. J. L. Cascales, and J. G. de la Torre, Macromolecules 26, 3851 (1993).

${ }^{32}$ A. Celani, A. Puliafito, and K. Turitsyn, Europhys. Lett. 70, 464 (2005).

${ }^{33}$ T. Liu, J. Chem. Phys. 90, 5826 (1989).

${ }^{34}$ J. S. Hur and E. S. G. Shaqfeh, J. Rheol. 44, 713 (2000).

${ }^{35}$ P. P. Jose and G. Szamel, J. Chem. Phys. 128, 224910 (2008).

${ }^{36}$ G.-L. He, R. Messina, H. Löwen, A. Kiriy, V. Bocharova, and M. Stamm, Soft Matter 5, 3014 (2009).

${ }^{37}$ J. K. G. Dhont, An Introduction to Dynamics of Colloids (Elsevier, Amsterdam, 1996).

${ }^{38}$ J. Rotne and S. Prager, J. Chem. Phys. 50, 4831 (1969).

${ }^{39}$ K. D. Knudsen, J. G. de la Torre, and A. Elgsaeter, Polymer 37, 1317 (1996).

${ }^{40}$ A. V. Lyulin, D. B. Adolf, and G. R. Davies, J. Chem. Phys. 111, 758 (1999).

${ }^{41}$ D. Petera and M. Muthukumar, J. Chem. Phys. 111, 7614 (1999).

${ }^{42}$ R. M. Jendrejack, J. J. de Pablo, and M. D. Graham, J. Chem. Phys. 116, 7752 (2002)

${ }^{43}$ C.-C. Hsieh and R. G. Larson, J. Rheol. 48, 995 (2004).

${ }^{44}$ S. Liu, B. Ashok, and M. Muthukumar, Polymer 45, 1383 (2004).

${ }^{45}$ R. Pamies, M. C. L. Martinez, J. G. H. Cifre, and J. G. de la Torre, Macromolecules 38, 1371 (2005).

${ }^{46}$ C. Sendner and R. R. Netz, EPL 81, 54006 (2008).

${ }^{47}$ C. Sendner and R. R. Netz, Eur. Phys. J. E 30, 75 (2009).

${ }^{48}$ Y. Zhang, A. Donev, T. Weisgraber, B. J. Alder, M. G. Graham, and J. J. de Pablo, J. Chem. Phys. 130, 234902 (2009).

${ }^{49}$ C. Pierleoni and J.-P. Ryckaert, Macromolecules 28, 5097 (1995).

${ }^{50}$ C. Aust, M. Kröger, and S. Hess, Macromolecules 32, 5660 (1999).

${ }^{51}$ Y. Gratton and G. W. Slater, Eur. Phys. J. E 17, 455 (2005).

${ }^{52}$ J. F. Ryder and J. M. Yeomans, J. Chem. Phys. 125, 194906 (2006).

${ }^{53}$ M. Ripoll, R. G. Winkler, and G. Gompper, Phys. Rev. Lett. 96, 188302 (2006).

${ }^{54}$ H. Kobayashi and R. Yamamoto, Phys. Rev. E 81, 041807 (2010).

${ }^{55}$ R. G. Winkler, Phys. Rev. Lett. 82, 1843 (1999).

${ }^{56}$ R. G. Winkler, S. Keller, and J. O. Rädler, Phys. Rev. E 73, 041919 (2006).

${ }^{57}$ E. P. Petrov, T. Ohrt, R. G. Winkler, and P. Schwille, Phys. Rev. Lett. 97, 258101 (2006).

${ }^{58}$ M. Hinczewski, X. Schlagberger, M. Rubinstein, O. Krichevsky, and R. R. Netz, Macromolecules 42, 860 (2009).

${ }^{59}$ M. Hinczewski and R. Netz, EPL 88, 18001 (2009)

${ }^{60}$ M. G. Bawendi and K. F. Freed, J. Chem. Phys. 83, 2491 (1985).

${ }^{61}$ S. M. Bhattacharjee and M. Muthukumar, J. Chem. Phys. 86, 411 (1987).

${ }^{62}$ J. B. Lagowski, J. Noolandi, and B. Nickel, J. Chem. Phys. 95, 1266 (1991).

${ }^{63}$ R. G. Winkler, P. Reineker, and L. Harnau, J. Chem. Phys. 101, 8119 (1994).

${ }^{64}$ B. Y. Ha and D. Thirumalai, J. Chem. Phys. 103, 9408 (1995).

${ }^{65}$ R. G. Winkler, J. Chem. Phys. 118, 2919 (2003).

${ }^{66}$ L. Harnau, R. G. Winkler, and P. Reineker, J. Chem. Phys. 102, 7750 (1995).

${ }^{67}$ L. Harnau, R. G. Winkler, and P. Reineker, J. Chem. Phys. 104, 6355 (1996).

${ }^{68}$ R. A. Harris and J. E. Hearst, J. Chem. Phys. 44, 2595 (1966).

${ }^{69}$ K. F. Freed, Adv. Chem. Phys. 22, 1 (1972).

${ }^{70}$ R. G. Winkler, L. Harnau, and P. Reineker, Macromol. Theory Simul. 6, 1007 (1997)

${ }^{71}$ R. G. Winkler, J. Chem. Phys. 127, 054904 (2007).

${ }^{72}$ H. Risken, The Fokker-Planck Equation (Springer, Berlin, 1989).

${ }^{73}$ R. G. Winkler and P. Reineker, Macromolecules 25, 6891 (1992).

${ }^{74}$ O. Kratky and G. Porod, Recl. Trav. Chim. Pays-Bas 68, 1106 (1949). 
${ }^{75}$ E. Farge and A. C. Maggs, Macromolecules 26, 5041 (1993).

${ }^{76}$ K. Kroy and E. Frey, Phys. Rev. E 55, 3092 (1997).

${ }^{77}$ B. H. Zimm, J. Chem. Phys. 24, 269 (1956)

${ }^{78}$ F. Ganazzoli, G. Allegra, E. Colombo, and M. D. Vitis, Macromolecules 28, 1076 (1995).

${ }^{79}$ C.-C. Huang, R. G. Winkler, G. Sutmann, and G. Gompper, Macromolecules, accepted for publication.
${ }^{80}$ J. S. Hur and E. S. G. Shaqfeh, J. Rheol. 45, 421 (2001).

${ }^{81}$ H. C. Öttinger, Phys. Rev. A 41, 4413 (1990).

${ }^{82}$ L. E. Wedgewood, J. Non-Newtonian Fluid Mech. 31, 127 (1989).

${ }^{83}$ T. T. Perkins, D. E. Smith, R. G. Larson, and S. Chu, Science 268, 83 (1995).

${ }^{84}$ D. E. Smith, T. T. Perkins, and S. Chu, Macromolecules 29, 1372 (1996).

${ }^{85}$ Y. Liu, Y. Jun, and V. Steinberg, Macromolecules 40, 2172 (2007). 